\title{
Topology Optimization Analysis of the Working Arm in the Slag Pot Carrier Based on Hyperworks
}

\author{
Shihuai Shi ${ }^{1, a}$, Ruifeng Guo ${ }^{1, b}$ and Yanhe $\mathrm{Li}^{1, \mathrm{c}}$ \\ ${ }^{1}$ School of Mechanical and Electrical Engineering, Xi'an University of Architecture and Technology, \\ Xi'an 710055, China \\ a1032694968@qq.com, b852370757@qq.com, c724728441@qq.com
}

Keywords: Slag Pot Carrier, Working Arm, Hypermesh, Topology optimization.

\begin{abstract}
Aiming at the problem of great mass and low material utilization coefficient of the working arm in a certain type of slag pot carrier, the static analysis and topology optimization analysis of the working arm were carried out. The finite element model of the working arm was established by using the pre-processing module of Hypermesh and its solution was solved. Post-processing was carried out by using the Hyperview module, and the stress- deformation diagram of the working arm under the working conditions of loading, transporting and pouringof slag potwere obtained. The Optistruct solver wasemployed to do thetopology optimization and a reasonable improvement scheme is proposed based on the optimization result.
\end{abstract}

\section{Introduction}

Slag pot carrier is a no-rail transport equipment used for transporting slag inthe steel plant[1]. This kind of vehicle sets loading, transporting, pouring of a slag pot and other functions in one[2], which is flexible, efficient, and not limited by route or loading points. However, the working arm has a large mass and a low material utilization coefficient, which not only increase the cost of production, but also is not very efficient for its operation.

Hyperworks software is the main product of Altair company, it is a complete CAE modeling, visualization, finite element analysis, structural optimization and process automation and other fields of products, always stands in the forefront of technology[3]. It has Hypermesh, Hyperview, Hypercrash, Optistruct, Hyperstudy, Radioss, etc in its rich product modules. In this paper, the HyperMesh, Optistructmodules wereemployed to deal with the pre-processing and the solution of the finite element model of the working arm. The post-processing was carried out by using the Hyperview module.

\section{The establishment and the pre-processing of the working arm}

The overall structure of slag pot carrier is shown in Fig 1.

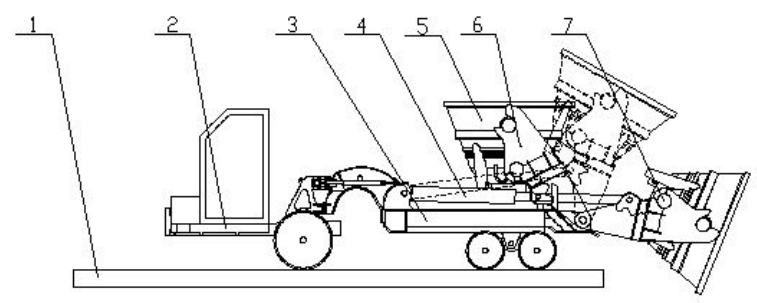

Fig. 1 The overall structure of slag pot carrier

1. ground 2. front frame 3. rear frame 4. hydro-cylinder

5. slag pot 6 . the working arm 7. the pulling cylinder

The model of the working arm established by using of Pro/E is shown in Fig 2. 


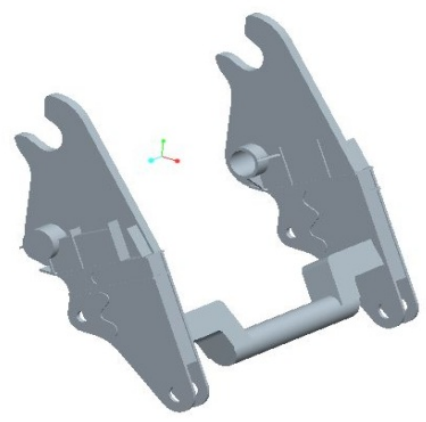

Fig. 2 The working arm model

The Pro/E model was introduced into the Hypermesh for pre-processing (in order to save computing resources, the $1 / 2$ model was taken for analysis). The working arm was divided into design space and non-design space by using the Geom panel in Hypermesh, and the non-design space contains the area connecting to the other parts of the carrier.

A tetrahedral element size of $15 \mathrm{~mm}$ was selected to mesh the working arm, and a RBE2 element was created to simulate the stress state of the pulling cylinder. 783887 elements and 196008 nodes were created after the mesh processing.

Material parameters of the working arm are as bellows:

Material type: Q345B

Elastic modulus: 2.1E5Mpa

Density: $7.85 \mathrm{E}-9 \mathrm{t} / \mathrm{mm}^{3}$

Poisson's ratio: 0.3 .

The dynamics simulation software Adams/viewwas used to do the dynamics simulation[4] of the working arm mechanism. The force of the slideway and the pulling cylinder under the working conditions of loading, transporting and pouring were obtained, as shown in Table 1.

Table 1 The force of the working arm(newton)

\begin{tabular}{ccc}
\hline Subcase & Slideway $(\mathrm{X}, \mathrm{Y}, \mathrm{Z})$ & Pulling cylinder(X, Y, Z) \\
\hline Loading & $(359470,175450,0)$ & $(0,0,0)$ \\
Transporting & $(0,-400000,0)$ & $(0,0,0)$ \\
Pouring & $(151871,201221,0)$ & $(149885,48893,0)$ \\
\hline
\end{tabular}

The constraints condition of loading and pouring conditions were: release the Z-axis rotation degree of freedom in the connection joint between the working arm and the rear frame and constraint the remaining 5 degrees of freedom; release the Z-axis rotation degree of freedom in the connection joint between the working arm and the hydro-cylinder and constraint the remaining 5 degrees of freedom; constraint the Z-displacement on the symmetrical plane of the working arm, and release the remaining 5 degrees of freedom.

The constraints condition of transporting condition were: release the Z-axis rotation degree of freedom in the connection joint between the working arm and the rear frame and constraint the remaining 5 degrees of freedom; constraint the Y-axis displacement in the connection between the working arm and the limit block on the rear frame and releasethe remaining 5 degrees of freedom; constraint the Z-displacement on the symmetrical plane of the working arm, and release the remaining 5 degrees of freedom.

The finite element model built is shown in Fig 3.

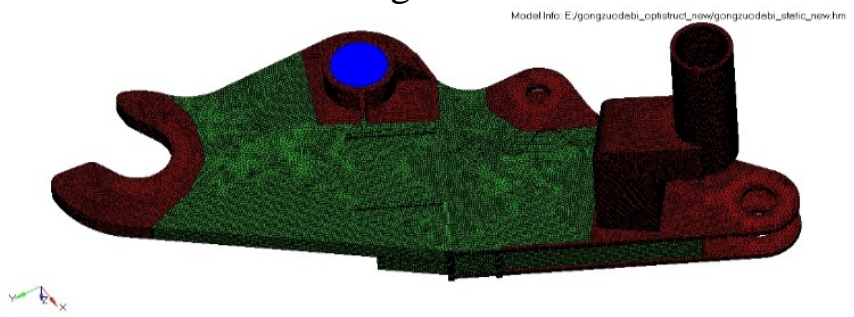

Fig. 3 The finite element model of the working arm 


\section{The finite element analysis and the topology optimization analysis}

Von Mise stress was selected to evaluate the stress intensity of the working arm according to the fourth strength theory [5]. Von Mise equivalent stress is expressed as:

$$
\sigma_{r}=\sqrt{\left[\left(\sigma_{1}-\sigma_{2}\right)^{2}+\left(\sigma_{2}-\sigma_{3}\right)^{2}+\left(\sigma_{31}-\sigma_{1}\right)^{2}\right] / 2}
$$

The strength condition is expressed as: $\sigma_{r} \leq[\sigma]$,where $\sigma_{1}, \sigma_{2}, \sigma_{3}$ are the first, second and third principal stress respectively, $\sigma_{r}$ is the equivalent stress and $[\sigma]$ is the allowable stress of material.

Safety factor was set to 2 , then $[\sigma]=\sigma_{s} / 2=172.5 \mathrm{MPa}$.

The finite element model was submitted to Optistruct for static analysis, and deformation and stress contours were obtained as shown in Fig 4 and Fig 5(the left is loading condition, the middle is transporting condition and the right is pouring condition).
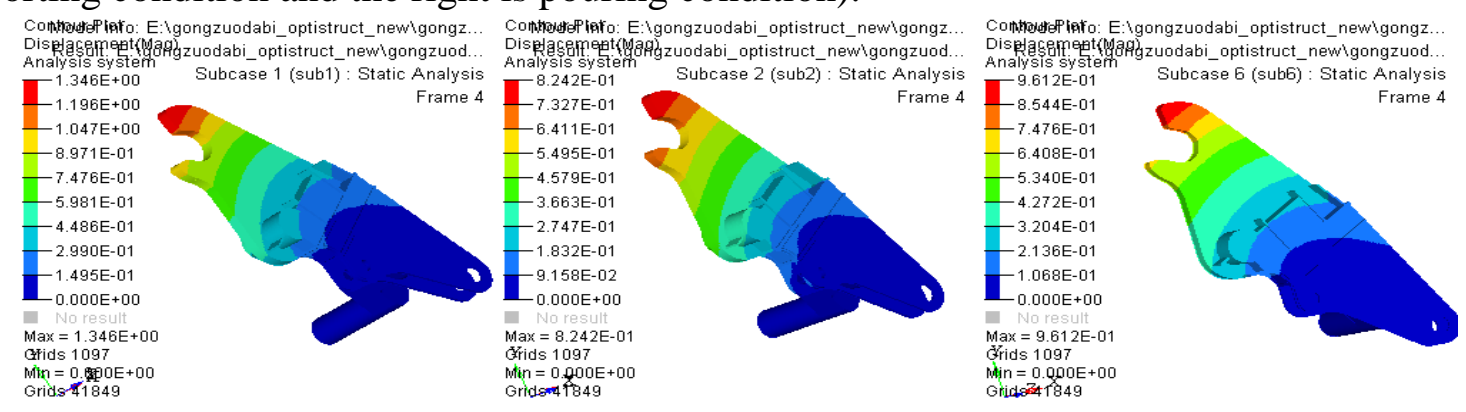

Fig. 4 Deformation contours
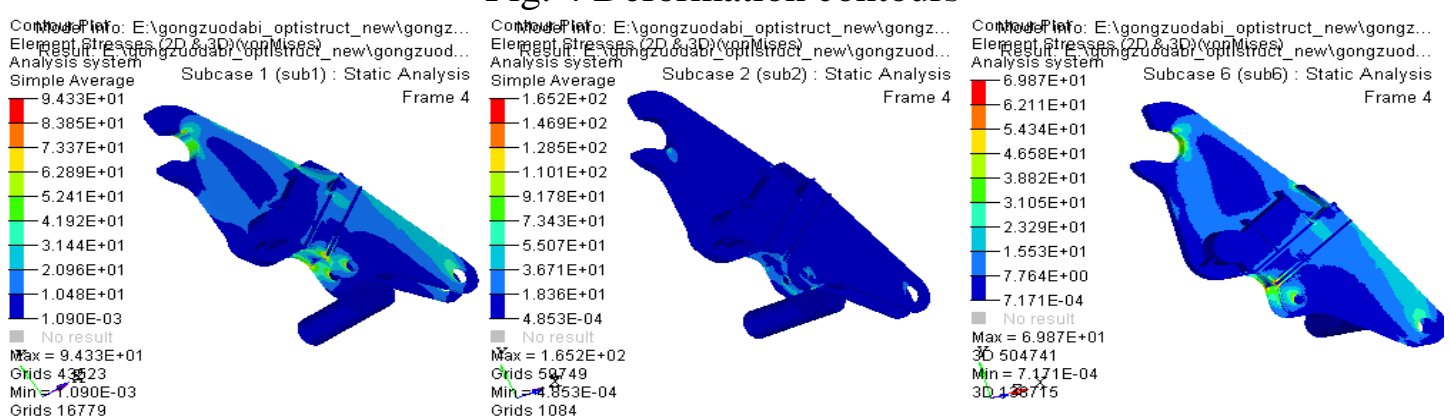

Fig. 5 Stress contours

The analysis results show that the maximum displacement is $1.346 \mathrm{~mm}$ which occurs in the loadingcondition; the maximum stress is $165.2 \mathrm{Mpa}$ (less than the allowable stress $172.5 \mathrm{Mpa}$ ), which occurs in the transporting conditions. It can be seen that the stress level of many areas is very low, which leads to a waste of material.The topology optimization of the working arm was used to raise the material utilization ratio.

In the topology optimization, if minimum mass (or minimum volume) was set as the optimization objective, most of the material in the model would be removed, which is obviously unreasonable. In this analysis, the minimum compliance (maximum stiffness) is set as the optimization objective, the volume fraction is set as the optimization constraint (upper bound is 0.3), and the variable density method [6] is adopted to carry out the topology optimization analysis by using Optistruct solver.

The optimization result is shown in Fig 6 when the element density is greater than 0.3. 

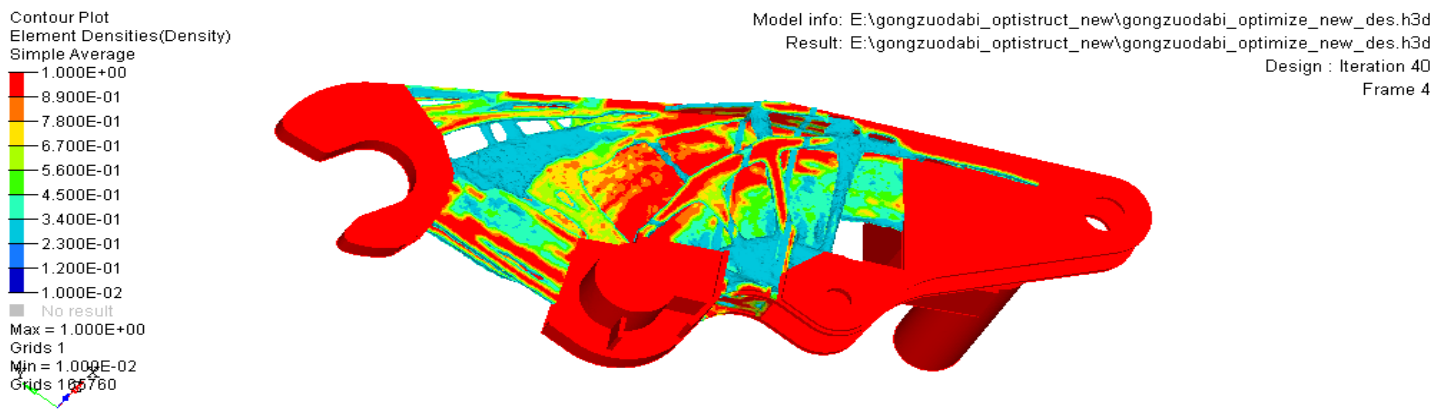

Fig. 6 Element density result contour

The topology optimization result after manufacturability process is shown in Figure 7.

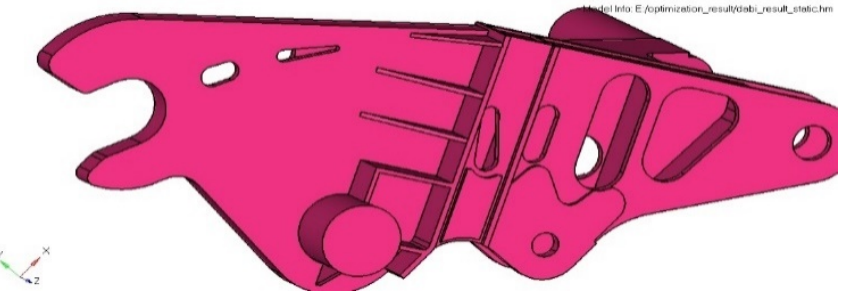

Fig. 7 The improved working arm model

Reanalysis the loading, transporting and pouring working conditions of the improved arm model. The results show that the maximum stress still appears in the transporting condition, but the value reducedto $130.8 \mathrm{Mpa}$;Compared with the analysis results of the original model, the maximum displacement of the improved model increases a little, but it is still in the acceptable range. The mass of the original model ( $1 / 2$ model) is 4.316tons, and it reduces to 3.656tons after topology optimization, the mass is reduced by $15.3 \%$, the improvement is very effective.

\section{Summary}

The finite element model was established by using of pre-processor Hypermesh. The material utilization coefficient was found at a low level through the finite element analysis of three working conditions of the working arm. The improved model was established after the topology optimization and manufacturability process. The finite element analysis shows that the new model well meets the working conditions while the mass reduces by $15.3 \%$, which shows the optimization is very effective.

\section{References}

[1] Ren Zhongli. The Development of Articulated Slag Transport Vehicle. Journal of Hubei University of Technology. Vol. 23(2008) No. 02, p. 30-31.

[2] Geng Huiliang, Ren Zhongli. One Type of Slag Pot Carrier Unloading With Large Angle. Journal of Hubei University of Technology. Vol. 25(2010) No. 04, p. 80-82.

[3] Wang Yudong, Jin Lei, Hong Qingquan, et al. HyperMesh\&Hyperview Application Skills and Advanced Examples. China Machine Press, 2012, p. 2-4.

[4] GuoWeidong, Li Shouzhong, Ma Lu. ADAMS 2013 Advanced Application Tutorial with Examples. China Machine Press, 2015, p. 3-12.

[5] Liu Hongwen. Mechanics of Materials. Higher Education Press, 2004, p. 243-246.

[6]Stolpe M, Svanberg K. An alternative interpolation scheme for minimum compliance. Structural Multidiscipline Optimization.Vol. 22(2001) No. 02, p. 116-124. 